\title{
Does size matter? Retrospective analysis of large gynecologic tumors
}

\author{
Tufan Öge ${ }^{1}$, Emel Öztürk², Ömer T. Yalçın \\ 1Department of Obstetrics and Gynecology, Eskişehir Osmangazi University School of Medicine, Eskişehir, Turkey \\ ${ }^{2}$ Clinic of Obstetrics and Gynecology, Beyhekim State Hospital, Konya, Turkey
}

\begin{abstract}
Objective: To evaluate the characteristics of patients who underwent surgery due to the presence of a large pelvic-abdominal mass over a 5 -year period in a university clinic.

Material and Methods: Among 3476 gynecologic operations, intraoperative findings were evaluated retrospectively. Uterine and/or adnexal masses smaller than $20 \mathrm{~cm}$ were excluded to refine "large" tumors and 74 patients with large tumors were enrolled in the study group. Demographic characteristics, intraoperative findings, and results of histopathologic examinations were recorded. Moreover, preoperative and intraoperative findings were compared among tumors with adnexal origin according to their final histopathologic results.

Results: The mean age of the patients was 46 years. The most common symptom was abdominal pain, as recorded in 38 (51.4\%) patients. Among all patients, 31 (41.9\%) had coexisting illness and 13 (17.6\%) had a history of surgery. The mean tumor diameter was 25.9 $\pm 8.6 \mathrm{~cm}(20-60)$ and $60(78.9 \%)$ tumors were of adnexal origin. The ratios of malignancy for large adnexal and uterine tumors were $34.4 \%$ and $12.5 \%$, respectively. When the large adnexal tumors were re-evaluated, the mean cancer antigen (CA) 125 level was significantly higher, and ascites was more frequently detected in malignant tumors $(\mathrm{p}<0.01)$ then in benign and borderline tumors.

Conclusion: Benign and borderline tumors are more common among large abdominopelvic masses, although the presence of ascites and elevated CA 125 may present malignancy in large gynecologic tumors. Further studies with larger sample sizes are needed to define the characteristics of large tumors and their malignant potentials. (J Turk Ger Gynecol Assoc 2017; 18: 195-9)
\end{abstract}

Keywords: Big gynecologic tumors, borderline tumors, leiomyoma, ovarian cysts

Received: 7 March, 2017 Accepted: 1 August, 2017

\section{Introduction}

Managing pelvic masses, which may result from benign or malignant conditions of gynecologic and non-gynecologic diseases, is routine daily practice for outpatient clinic gynecologists. However, due to its rarity, large-sized pelvic tumors that reach the upper abdomen may sometimes be confusing for physicians $(1,2)$. The size of the adnexal mass is one of the important factors in making decisions for the clinical management, and therefore some indexes have to be taken into account (3-7). Although "giant", "huge," and "large" tumor descriptions are not very clear, and the cut-off sizes of these explanations are inconclusive, gynecologists and oncologists can diagnose large pelvic-abdominal masses that require treatment.
To our knowledge, although the English literature consists of case reports including large gynecologic tumors of ovarian, tubal, and uterine origin, there is lack of data regarding a series of large tumors (8-10). From this point of view, we aimed to evaluate the characteristics of patients who were diagnosed as having and underwent surgery for large pelvic-abdominal masses over a 5 -year period in a university clinic.

\section{Material and Methods}

After obtaining approval from the local ethics committee, the surgical and pathologic reports of patients who underwent surgical procedures due to suspected adnexal masses between 2011 and 2016 were retrospectively reviewed in our university clinic.

All patients underwent detailed pelvic examination, ultrasonographic evaluation and computerized tomography 
or magnetic resonance imaging (MRI) if needed. Thereafter, all patients were evaluated by the tumor board and surgery indications were approved. After preoperative medical evaluations, all patients underwent laparotomy through a midline incision. Adnexal masses with suspected malignancies were sent for frozen section. According to frozen section histopathologic evaluations, patients with malignant masses underwent debulking surgery, and patients with benign disease underwent conservative surgery or total hysterectomy and salpingo-oophorectomy. Among 3476 gynecologic operations, intraoperative findings were evaluated and uterine and/or adnexal masses smaller than $20 \mathrm{~cm}$ were excluded to refine "large" tumors, and 74 patients with large tumors were enrolled in the study group. A flow chart of the patients is shown in Figure 1.

Demographic characteristics including age, gravidity, parity, medical history, symptoms, physical and pelvic examination, ultrasonographic evaluations, cancer antigen (CA) 125 levels, intraoperative findings, and results of the histopathologic examinations of the patients were recorded. In addition, preoperative and intraoperative findings were compared among tumors with adnexal origin according to their final histopathologic results.

Statistical analysis of the collected data was performed using IBM Statistical Package for Social Sciences (SPSS) 23.0 software. The normality of distribution was checked initially using Shapiro-Wilk's test and parametric or non-parametric tests were applied to data with normal or non-normal distribution, respectively. One-way analysis of variance (ANOVA) with Tukey's honest significant difference post hoc test and KruskalWallis (ANOVA on Ranks) tests with Dunn's post hoc tests were applied to determine the differences among benign, borderline, and malignant adnexal masses. Chi-square tests were applied for categorical variables. The results are expressed as mean \pm standard deviation and median (interquartile range $\mathrm{Q} 1$ and $\mathrm{Q} 3)$; p values $<0.05$ were considered statistically significant.

\section{Results}

The mean age of the patients was 47 years. Of the patients, $54.1 \%(n=40)$ were premenopausal and $45.9 \%(n=40)$ were postmenopausal. Two of the premenopausal patients were adolescents. The most common symptom was abdominal pain, which was recorded in 38 (51.4\%) patients. Among all patients, 31 (41.9\%) had coexisting illness and 13 (17.6\%) had a history of surgery. The patients' demographic characteristics, preoperative CA 125, and hemoglobin levels are summarized

\section{Table 1. Patient characteristics}

\begin{tabular}{|l|l|}
\hline Age, (years) & $47.3 \pm 14.6$ \\
\hline Gravida, (n) & $2.5 \pm 2.3$ \\
\hline Parity, (n) & $1.8 \pm 1.5$ \\
\hline Symptoms, (n) & $38(51.4 \%)$ \\
Abdominal pain & $16(21.6 \%)$ \\
Abdominal distension & $15(19 \%)$ \\
Abdominal mass & $5(6 \%)$ \\
Menometrorrhagia & \\
\hline Coexisting illness, (n) & $16(21.6 \%)$ \\
Hypertension & $6(8 \%)$ \\
Hypothyroidism & $5(6 \%)$ \\
Diabetes mellitus & $4(5 \%)$ \\
Asthma bronchial & \\
\hline History of surgery (n) & $7(9 \%)$ \\
Cesarean section & $6(8 \%)$ \\
Other & $98.4 \pm 165$ \\
\hline CA 125 (U/mL) & $12.3 \pm 1.8$ \\
\hline Preoperative hemoglobin (g/dL) & \\
\hline Data are given as mean \pm standard deviation or No (\%) & \\
\hline
\end{tabular}

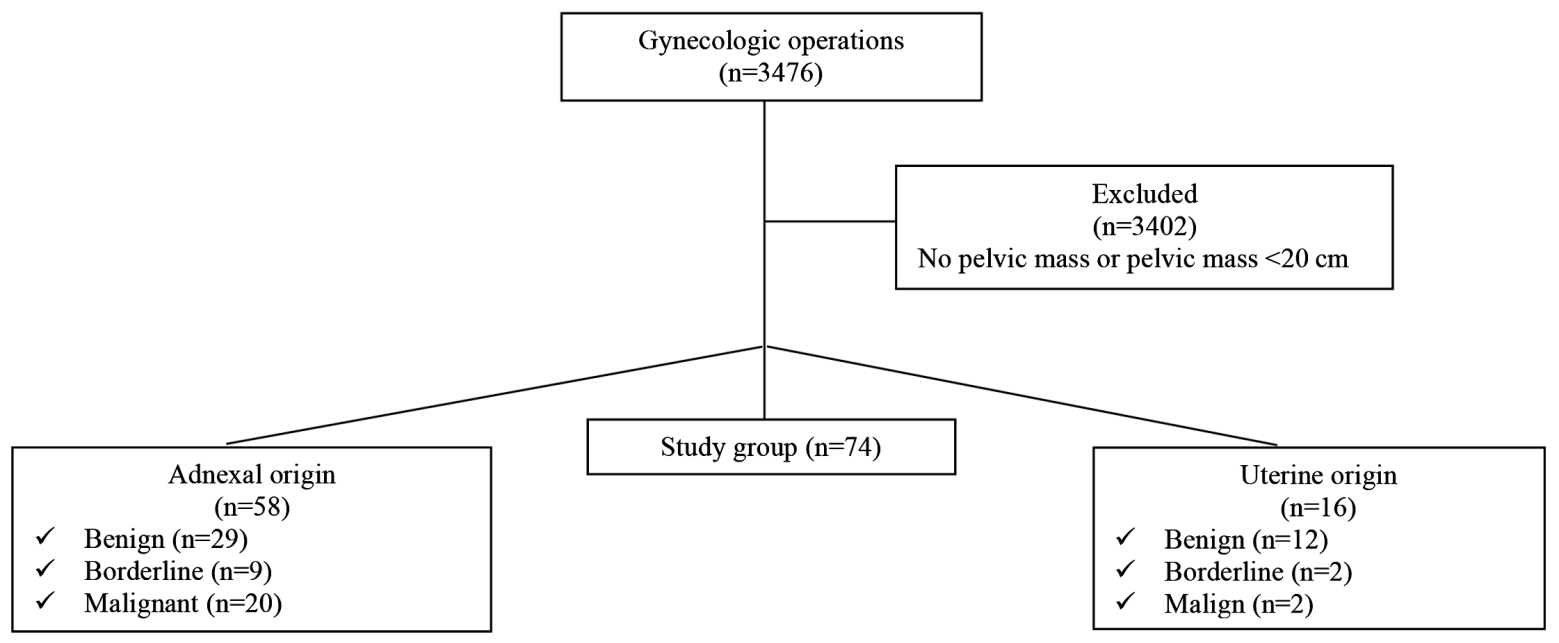

Figure 1. Flow chart of the patients 
in Table 1. According to the operation findings, the mean tumor diameter was $25.9 \pm 8.6 \mathrm{~cm}(20-60)$ and 60 (78.9\%) of the tumors were of adnexal origin. During the operations, adhesiolysis was performed in 30 (40.5\%) operations, 4 small bowel lacerations, 1 bladder, and 1 sigmoid perforation occurred, all were repaired in the same session. Exploration findings during laparotomy are summarized in Table 2 . Among 74 operations, 58 of 60 adnexal masses were sent for frozen section analysis. None of the large tumors of uterine origin $(n=14)$ were evaluated using frozen section analysis. Frozen section examination revealed benign, borderline, and malignant tumors in 29 (50\%), 9 (15.5\%), and 20 (34.4\%) patients with adnexal masses, respectively. Histopathologic examination revealed leiomyosarcoma in 2 (12.5\%) of 16 patients with gynecologic tumors of uterine origin. When patients were classified according to menopausal status, final histopathologic examinations revealed malignancy in 13 of the $34(38.2 \%)$ menopausal patients, and 9 of the $40(22.5 \%)$ pre-menopausal patients. Moreover, 2 of the pre-menopausal patients were adolescents and their evaluations were regarded as benign disease. The ratios of malignancy for large adnexal and uterine tumors were $34.4 \%$ and $12.5 \%$, respectively. Detailed final histopathologic examination distributions are shown in Table 3.

When the adnexal big tumors were re-evaluated, the mean CA 125 level was significantly higher, and ascites was more frequently detected in malignant tumors $(p<0.01)$ than in benign and borderline tumors. The characteristics of the large adnexal tumors according to final histopathologic results are summarized in Table 4.

\section{Discussion}

In the present study, we aimed to evaluate and share our experience of large abdominopelvic tumors that underwent

Table 2. Exploration findings during laparotomy

\begin{tabular}{|l|l|}
\hline Site of tumor, (n) & $60(81.1 \%)$ \\
Adnexa & $14(18.9 \%)$ \\
\hline Uterus & $25.9 \pm 8.6$ \\
(miameter of tumor, (cm) & $(20-60)$ \\
\hline Weight of tumor, (g) & $5555 \pm 2241$ \\
(minimum - maximum) & $(3500-14600)$ \\
\hline Presence of ascites, (n) & \\
Yes & $57(77 \%)$ \\
No & $17(23 \%)$ \\
\hline Frozen section results, (n) & $29(50 \%)$ \\
Benign & $9(15.5 \%)$ \\
Borderline & $20(34.5 \%)$ \\
\hline Malignant & \\
\hline Data are given as mean \pm standard deviation or No (\%) \\
\hline
\end{tabular}

surgery in a university gynecology clinic. Of the tumors with adnexal and uterine origin, $34.4 \%$ and $12.5 \%$ were found to be malignant, and $15.5 \%$ and $12 \%$ of tumors were diagnosed as borderline ovarian and uterine smooth muscle tumors of uncertain malignant potential, respectively. The mean size and weight of tumor were not statistically different between benign, borderline, and malignant large tumors; however, CA 125 was found to be elevated, and the presence of ascites was significantly detected in large malignant tumors.

One of the limitations of this study is its retrospective design. All operation notes were evaluated but there may still have been

Table 3. Final histopathologic results

\begin{tabular}{|l|l|}
\hline & n (\%) \\
\hline Benign & \\
Mucinous cystadenoma/fibroma & $14(18.9 \%)$ \\
Simple/functional cysts & $4(5.4 \%)$ \\
Fibroma & $4(5.4 \%)$ \\
Endometrioma & $3(4 \%)$ \\
Serous cystadenoma/fibroma & $2(2.7 \%)$ \\
Teratoma & $2(2.7 \%)$ \\
Leiomyoma & $12(16.3 \%)$ \\
\hline Borderline & \\
Borderline mucinous cystadenoma & $7(9.5 \%)$ \\
Borderline serous cystadenoma & $2(2.7 \%)$ \\
STUMP* & $2(2.7 \%)$ \\
\hline Malignant & \\
Mucinous adenocarcinoma & $6(8.2 \%)$ \\
Serous adenocarcinoma & $5(6.7 \%)$ \\
Endometrioid adenocarcinoma & $5(6.7 \%)$ \\
Clear cell carcinoma & $2(2.7 \%)$ \\
Metastatic carcinoma & $2(2.7 \%)$ \\
Leiomyosarcoma & $2(2.7 \%)$ \\
\hline Total & $\mathbf{7 4 ( 1 0 0 \% )}$ \\
\hline STUMP: Smooth muscle tumors of uncertain malignant potential \\
\hline
\end{tabular}

Table 4. Characteristics of big tumors with adnexal origin according to final histopathologic results

\begin{tabular}{|l|l|l|l|l|}
\hline & Benign & Borderline & Malignant & p \\
\hline Age, (years) & $45.8 \pm 17.4$ & $48.7 \pm 15.3$ & $50.3 \pm 13.3$ & $>0.05$ \\
\hline $\begin{array}{l}\text { CA 125 } \\
\text { (U/mL) }\end{array}$ & $28(17-43)$ & $18(17-27)$ & $122(42-294)$ & $<\mathbf{0 . 0 1}$ \\
\hline Hb (g/dL) & $12.5 \pm 1.7$ & $12.3 \pm 1.6$ & $12.1 \pm 1.6$ & $>0.05$ \\
\hline $\begin{array}{l}\text { Presence of } \\
\text { ascites, (n) }\end{array}$ & $3(19 \%)$ & $2(13 \%)$ & $11(68 \%)$ & $<\mathbf{0 . 0 1}$ \\
\hline $\begin{array}{l}\text { Diameter of } \\
\text { tumor, (cm) }\end{array}$ & $25(20-35)$ & $20(20-26)$ & $25(20-30)$ & $>0.05$ \\
\hline $\begin{array}{l}\text { Weight of } \\
\text { tumor, (g) }\end{array}$ & $\begin{array}{l}5000 \\
(4200-6700)\end{array}$ & $\begin{array}{l}6000 \\
(4650-7200)\end{array}$ & $\begin{array}{l}4550 \\
(4100-5000)\end{array}$ & $>0.05$ \\
\hline $\begin{array}{l}\text { CA 125: Cancer antigen 125; Hb: Hemoglobin } \\
\text { Data are given as mean } \pm \text { standard deviation for normally distributed } \\
\text { variables, median (25\%-75\%) for non-normally distributed variables, or } \\
\text { No (\%); a value <0.05 was considered statistically significant }\end{array}$ \\
\hline
\end{tabular}


patients who did not undergo surgery or were lost before the operation. Moreover, our cut-off limit may be questionable; however, to our knowledge, there is no consensus for the size of tumors to call them large, huge, or giant. Therefore, after searching the literature and our patients' charts, we set out cut-off level for "large" tumors at $20 \mathrm{~cm}$, and evaluated the operative characteristics of the patients in order to share our experience of large tumors. Also, we included all uterine and adnexal tumors in our study because we aimed to evaluate all large-sized tumors. Before surgery, all patients underwent ultrasonography and there was no question as to whether the origin was uterus or adnexa. Another limitation of this study is the sample size, even though large tumors are not very common. Our sample size was very small for large tumors with uterine origin; therefore, we only compared the operative characteristics of patients with large adnexal tumors.

During gynecology and gynecologic oncology practice, physicians usually diagnose adnexal masses and the most important issue it to exclude malignancy during the management. Ultrasonographic evaluation, menopausal status, and tumor markers such as CA 125 and HE4 are important predictors for malignancy $(4,11)$. Moreover, the presence of a multilocular cystic lesions, solid areas, bilateral lesions, ascites, and intra-abdominal metastases are also known to be important parameters during ultrasonographic evaluations. These features are known to be important morphologic features of adnexal masses. In addition, unilocular tumors, smooth multilocular tumors and no intra-tumoral blood flow in color or power Doppler are simple rules to predict benign disease, whereas irregular solid tumor, ascites, at least 4 papillary projections, and strong intra-tumoral blood flow in color or power Doppler may predict malignancy (12). Efforts are ongoing to standardize ultrasonographic evaluations for optimal patient management (13). For larger tumors, computed tomography (CT) or MRI may be required to determine the origin of the tumor. In the present study, 64 (86.4\%) patients were evaluated using CT or MRI for the differential diagnose. MRI was preferred especially for suspected masses of uterine origin in 10 (13.5\%) patients, whereas 54 (72.9\%) patients were evaluated using CT to discriminate the origin of the tumor, possible metastasis, and predict optimal cytoreduction.

In addition, some scoring systems such as the Rajavithi-Ovarian Cancer Predictive Score [risk of ovarian malignancy algorithm and risk of malignancy index (RMI)] have been introduced to discriminate benign and malignant cases $(3,4,6)$. RMI is the one of the most common methods using the knowledge of menopausal status (M), ultrasound findings (U), and the serum CA 125 level, and is calculated as $\mathrm{M} \times \mathrm{U} \times \mathrm{CA} 125$; (a total ultrasound score of 0 yields $U=0$, a score of 1 yields $U=1$, and a score of 2 yields $U=3$. Premenopausal status yields $M=1$, and
post-M yields $M=3$. The serum level of CA 125 is applied directly to the calculation $(6,7)$. RMI is then developed as RMI 1, RMI 2, and RMI 3 using the same formula, but scoring differently, and RMI 4 where size (S) is taken into account in a formula as $\mathrm{S} \times$ $\mathrm{M} \times \mathrm{U} \times \mathrm{CA} 125$. RMI 4 takes tumor size $<7 \mathrm{~cm}$ as $\mathrm{S}=0$, and $\geq 7 \mathrm{~cm}$ as $\mathrm{S}=2$ in the formula, and was introduced to be more reliable than RMI 1, RMI 2, and RMI 3. However, the diagnostic accuracy is still inconclusive in large tumors and new studies are needed to evaluate this issue.

Although there are some protocols or indexes for the management of adnexal masses, frozen section analysis is usually mandatory for large tumors. Large tumors typically require a midline incision reaching the upper abdomen, and need extra care during operations, but the role of minimally invasive surgery cannot be ignored. In some studies, laparoscopy was suggested a feasible and safe treatment for women with large ovarian cysts with proper patient selection (14-16). However, surgeons should carefully consider the potential risk of malignancy in such patients, and surgeon experience may still be a limitation with large tumors.

Mucinous tumors are more likely present in large masses averaging 16 to $30 \mathrm{~cm}$ in diameter $(17,18)$. A retrospective study evaluating mucinous borderline tumors reported the median tumor size as $20 \mathrm{com}$ (range, $4-40 \mathrm{~cm}$ ) (19). In the present study, of the 9 borderline tumors, 7 (77\%) were mucinous, the mean diameter was $26 \mathrm{~cm}$. Although mucinous ovarian cancer is an uncommon subtype of malignant ovarian tumors and accounts for approximately $5 \%$ to $10 \%$ of ovarian carcinomas, we found that the most frequent histologic type was mucinous adenocarcinoma. This finding may be due to our selection criteria, only choosing masses $20 \mathrm{~cm}$ and above.

To conclude, physicians should be aware of the malignancy potential and plan the optimal surgical team and procedure because large gynecologic tumors require surgical treatment. Benign and borderline tumors are more common among large abdominopelvic masses although the presence of ascites and elevated CA 125 may present malignancy in large gynecologic tumors. Further studies with larger sample sizes are needed to define the characteristics of large tumors and their malignant potentials.

Ethics Committee Approval: Ethics committee approval was received for this study from the ethics committee of Eskişehir Osmangazi University School of Medicine (No: 52).

Informed Consent: Written informed consent was obtained from patients who participated in this study.

Peer-review: Externally peer-reviewed.

Author Contributions: Concept - T.Ö., E.Ö., Ö.T.Y.; Design T.Ö., E.Ö., Ö.T.Y.; Supervision - T.Ö., Ö.T.Y.; Materials - T.Ö., E.Ö.; 
Data Collection and/or Processing - T.Ö., E.Ö.; Analysis and/or Interpretation - T.Ö., E.Ö.; Literature Review - T.Ö., E.Ö., Ö.T.Y.; Writer - T.Ö., E.Ö., Ö.T.Y.; Critical Review - T.Ö., E.Ö., Ö.T.Y.

\section{Conflict of Interest: No conflict of interest is declared by the} authors.

Financial Disclosure: The authors declared that this study received no financial support.

\section{References}

1. Cerruto E, Sudano MC, Ettore C, La Greca G, La Greca MG. Difficult diagnosis of hemoperitoneum in a patient with a pelvic mass of large size. Int J Surg Case Rep 2016; 26: 197-8.

2. Shiber LD, Gregory EJ, Gaskins JT, Biscette SM. Adnexal masses requiring reoperation in women with previous hysterectomy with or without adnexectomy. Eur J Obstet Gynecol Reprod Biol 2016; 200: 123-7.

3. Yanaranop M, Tiyayon J, Siricharoenthai S, Nakrangsee S, Thinkhamrop B. Rajavithi-ovarian cancer predictive score (R-OPS): A new scoring system for predicting ovarian malignancy in women presenting with a pelvic mass. Gynecol Oncol 2016; 141: 479-84.

4. Romagnolo C, Leon AE, Fabricio ASC, Taborelli M, Polesel J, Del Pup L, et al. HE4, CA125 and risk of ovarian malignancy algorithm (ROMA) as diagnostic tools for ovarian cancer in patients with a pelvic mass: An Italian multicenter study. Gynecol Oncol 2016; 141: 303-11.

5. Sinha A. Retrospective analysis of suspicious pelvic masses using the Pelvic Mass Index: the Cardiff experience [MSc dissertation]. UK: Cardiff University; 2012. https://www.ncbi.nlm.nih.gov/pubm ed/?term $=$ Retrospective + analysis + of + suspicious + pelvic + mas ses + using + the + Pelvic + Mass + Index $\% 3 \mathrm{~A}+$ the + Cardiff + experi ence

6. Tingulstad S, Hagen B, Skjeldestad FE, Onsrud M, Kiserud T, Halvorsen T, et al. Evaluation of a risk of malignancy index based on serum CA-125, ultrasound findings and menopausal status in the pre-operative diagnosis of pelvic masses. Br J Obstet Gynaecol 1996; 103: 826-31
7. Yamamoto Y, Yamada R, Oguri H, Maeda N, Fukaya T. Comparison of four malignancy risk indices in the preoperative evaluation of patients with pelvic masses. Eur J Obstet Gynecol Reprod Biol 2009; 144: 163-7.

8. Katke RD. Giant mucinous cystadenocarcinoma of ovary: A case report and review of literature. J Midlife Health 2016; 7: 41-4.

9. Feng L, Liu Y, Liu H, Chen X. Chronic isolated fallopian tube torsion associated with huge hydrosalpinx and hemosalpinx in a postmenopausal woman: a case report and brief review. Eur J Obstet Gynecol Reprod Biol 2012; 164: 235-6.

10. Steward RG, Denhartog HW, Katz AR. Giant uterine leiomyomata. Fertil Steril 2011; 95: 1121.

11. Campos C, Sarian LO, Jales RM, Hartman C, Araújo KG, Pitta D, et al. Performance of the Risk of Malignancy Index for Discriminating Malignant Tumors in Women with Adnexal Masses. J Ultrasound Med 2016; 35: 143-52.

12. Timmerman D, Testa AC, Bourne T, Ameye L, Jurkovic D, Van Holsbeke C, et al. Simple ultrasound-based rules for the diagnosis of ovarian cancer. Ultrasound Obstet Gynecol 2008; 31: 681-90.

13. Timmerman D, Van Calster B, Testa A, Savelli L, Fischerova D, Froyman W, et al. Predicting the risk of malignancy in adnexal masses based on the Simple Rules from the International Ovarian Tumor Analysis group. Am J Obstet Gynecol 2016; 214: 424-37.

14. Vlahos NF, Iavazzo C, Marcopoulos MC, Alamanou A, Kouiroukidou P, Chatzidakis V, et al. Laparoscopic management of large ovarian cysts. Surg Innov 2012; 19: 370-4.

15. Hong JH, Choi JS, Lee JH, Son CE, Jeon SW, Bae JW. Laparoscopic management of large ovarian tumors: clinical tips for overcoming common concerns. J Obstet Gynaecol Res 2012; 38: 9-15.

16. Lim S, Lee KB, Chon SJ, Park CY. Is tumor size the limiting factor in a laparoscopic management for large ovarian cysts? Arch Gynecol Obstet 2012; 286: 1227-32.

17. Ledermann JA, Luvero D, Shafer A, O'Connor D, Mangili G, Friedlander M, et al. Gynecologic Cancer InterGroup (GCIG) consensus review for mucinous ovarian carcinoma. Int J Gynecol Cancer 2014; 24: 14-9.

18. Kelemen LE, Köbel M. Mucinous carcinomas of the ovary and colorectum: different organ, same dilemma. Lancet Oncol 2011; 12: $1071-80$.

19. Cömert DK, Üreyen I, Karalok A, Taşçı T, Türkmen O, Öcalan R, et al. Mucinous borderline ovarian tumors: Analysis of 75 patients from a single center. J Turk Ger Gynecol Assoc 2016; 17: 96-100. 